\title{
D-branes and Creation of Strings
}

\author{
Igor R. Klebanov ${ }^{\mathrm{a}}$ \\ a Joseph Henry Laboratories \\ Princeton University \\ Princeton, New Jersey 08544
}

\begin{abstract}
We review two types of D-branes processes where open strings are created. In the first type, a closed string incident on a collection of D-branes is converted into a number of open strings running along them. For the case of threebranes we compare the leading absorption rate with that in semiclassical gravity, and find exact agreement. A supersymmetric non-renormalization theorem guarantees that this agreement survives all corrections in powers of the string coupling times the number of branes. The second type of process is creation of stretched open strings by crossing D-branes. We show that this is possible whenever a $p$-brane passes through an $(8-p)$-brane positioned orthogonally to it. The extra attractive force exerted by the stretched open string is crucial for finding that the net force cancels in this BPS system.
\end{abstract}

\section{Introduction}

The Dirichlet branes (D-branes) [1]2] are a remarkable window into non-perturbative string theory. Their dynamics is neatly described by open strings whose end-points are free to move only along a $p$-dimensional hyperplane, the center of the Dirichlet $p$-brane. Since they are exchanged with the elementary strings under nonperturbative duality symmetries, the D-brane degrees of freedom are indispensable for the overall consistency of the theory. There are nice reviews explaining the physics of D-branes and their applications to string dualities [3].

While D-branes are non-perturbative objects whose tensions scale as $1 / g_{\text {str }}$, their interactions with closed strings are tractable in perturbation theory [4]. An example of such an interaction is scattering of a massless closed string off a Dbrane, which may be used to measure the D-brane form factors [5]. The leading order amplitudes of this type involve two bulk vertex operators on a disk and were calculated in [5, 7.4.

Another example is absorption of a closed string accompanied by production of some number of open strings moving along the D-brane. For general incoming energy, the lowest order in perturbation theory involves two open strings in the final state. The necessary amplitudes, which involve one bulk and two boundary vertex operators, were calculated explicitly in [8, 8 ]. While at high incident energy they were found to falloff exponentially, which indicates growth in the effective thickness of a D-brane, at low energies these amplitudes are in full agreement with the effective actions of the Dirac-Born-Infeld type [1, 3]. Thus, the low-energy behavior of the absorption cross-sections for massless particles may be reliably determined from the world volume effective actions of the D-branes. It is of obvious interest to compare the results with the absorption crosssections by the $p$-brane solutions in low-energy supergravity. This was done in [9, 10] and will be reviewed in section 2. For the threebrane, which is the only non-singular D-brane solution, a perfect agreement of the leading cross-sections was found [9, 10]. The higher order processes where $\ell+2$ open strings are produced by an incoming closed string turn out to be counterparts of the semiclassical absorption in the $\ell$-th partial wave. Thus, interesting comparisons can also be made for the higher partial waves. We also compare the semiclassical and the world volume absorption cross-sections for the twobrane and the fivebrane of M-theory, and find agreement in the scaling with the energy. The comparison of the coefficients, however, is impeded by the lack of information about the world volume theory of multiple 
M-branes.

In section 3 we move on to another aspect of the D-brane physics. We study the force balance between orthogonally positioned $p$-brane and $(8-p)$-brane. The force due to graviton and dilaton exchange is repulsive in this case. We identify the attractive force that balances this repulsion as due to one-half of a fundamental string stretched between the branes [11]. If the $p$-brane is initially to the left of the $(8-p)$-brane and the half-string points away from the $p$-brane then, after it crosses to the right, we find that the halfstring points toward the $p$-brane. This may be interpreted as creation of one fundamental string directed from the $(8-p)$-brane to the $p$-brane. We show this directly from the structure of the Chern-Simons terms in the D-brane effective actions. We also discuss the effect of string creation on the 0-brane quantum mechanics in the type I' theory. The creation of a fundamental string is related by U-duality to the creation of a 3-brane discussed by Hanany and Witten [12]. Both processes have a common origin in M-theory: as two M5-branes with one common direction cross, a M2-brane stretched between them is created.

\section{World Volume Approach to Absorption by Non-dilatonic Branes}

Extremal black holes with non-vanishing horizon area may be embedded into string theory or M-theory using intersecting $p$-branes 13 20]. These configurations are useful for a microscopic interpretation of the Bekenstein-Hawking entropy. The dependence of the entropy on the charges and the non-extremality parameter suggests a connection with $1+1$ dimensional conformal field theory. This 'effective string' is essentially the intersection of the $p$-branes, but its winding number grows as the product of the numbers of the branes involved in the intersection [21,19]. Therefore, the energy of the lowest excitation scales as the inverse of this product, in agreement with semiclassical considerations [21]. Thus, the embedding of black holes into string or M-theory gives an appealing picture of their lowest-energy excitations.

Calculations of emission and absorption rates
15,22 35 provide further tests of the 'effective string' models of $D=5$ black holes with three charges and of $D=4$ black holes with four charges. For minimally coupled scalars the functional dependence of the greybody factors on the frequency agrees exactly with semiclassical gravity, providing a highly non-trivial verification of the effective string idea [25,26]. Similar successes have been achieved for certain nonminimally coupled scalars, which were shown to couple to higher dimension operators on the effective string. For instance, the 'fixed' scalars [36] were shown to couple to operators of dimension $(2,2)$ [27], while the 'intermediate' scalars [32] to operators of dimension $(2,1)$ and $(1,2)$. Unfortunately, there is little understanding of the 'effective string' from first principles, and some of the more sensitive tests reveal this deficiency. For instance, the semiclassical gravity calculations of the 'fixed' scalar absorption rates for general black hole charges reveal a gap in our understanding of higher dimension operators 31]. A similar problem occurs when one attempts a detailed effective string interpretation of the higher partial waves of a minimally coupled scalar [33, 34]. Even the s-wave absorption by black holes with general charges is complex enough that it is not reproduced by the simplest effective string model [28,35]. These difficulties by no means invalidate the general qualitative picture, but they do pose some interesting challenges. We feel that, to gain insight into the general relation between gravity and Yang-Mills theory, it is useful to study, in addition to the intersecting branes, the simpler configurations which involve parallel branes only. Apart from their intrinsic interest, such configurations are also relevant to Schwarzschild black holes in Matrix theory [37], as was recently explained in [38,39].

A microscopic interpretation of the entropy of near-extremal $p$-branes was first explored in [40,41]. It was found that the scaling of the Bekenstein-Hawking entropy with the temperature agrees with that for a massless gas in $p$ dimensions only for the 'non-dilatonic $p$-branes': namely, the self-dual 3-brane of the type IIB theory, and the 2- and 5-branes of M-theory. In 42 a way of reconciling the differing scalings for the 
dilatonic branes was proposed. According to this 'correspondence principle' 43,42], the string theory and the semiclassical gravity descriptions are in general expected to match only at a special value of the temperature, which corresponds to the horizon curvature comparable to the string scale.17 In [42] it was shown that, in all known cases, the stringy and the Bekenstein-Hawking entropies match at this point up to factors of order 1. Part of the ambiguity in this factor comes from knowing the matching point only approximately. However, for the non-dilatonic branes this ambiguity is absent: the matching can be achieved at any scale because the stringy and the semiclassical entropies have identical scalings with temperature. This still leaves a relative factor of $4 / 3$ for the 3 -brane entropy 40 which, we hope, will eventually find an exact explanation in terms of the strongly coupled world volume theory.

The non-dilatonic branes have a number of special properties. A notable property of their extremal metrics is that the transverse part of the geometry is non-singular: instead of a singularity we find an infinitely long throat whose radius grows with the charge (the vanishing of the horizon area is due to the longitudinal contraction). Thus, for a large number $N$ of coincident branes, the curvature may be made arbitrarily small in Planck units. For instance, for $N$ D3-branes, the curvature is bounded by a quantity of order

$\frac{1}{\sqrt{N \kappa_{10}}} \sim \frac{1}{\alpha^{\prime} \sqrt{N g_{\mathrm{str}}}}$.

Thus, to suppress the string scale corrections to the classical metric, we need to take the limit $N g_{\text {str }} \rightarrow \infty$.

The tensions of non-dilatonic branes depend on $g_{\text {str }}$ and $\alpha^{\prime}$ only through the gravitational constant $\kappa$ in the appropriate dimension, which is also the only scale present in the semiclassical description. Indeed, the D3-brane tension is

$T_{(3)}=\frac{\sqrt{\pi}}{\kappa_{10}}$,

\footnotetext{
${ }^{1}$ For $N$ parallel D-branes, $N g_{\text {str }}$ is of order 1 at the match-
} ing point 42 . the M2-brane tension is

$T_{(2)}=\left(2 \pi^{2} \kappa_{11}^{-2}\right)^{1 / 3}$,

and the M5-brane tension is

$T_{(5)}=\left(\frac{\pi}{2 \kappa_{11}^{4}}\right)^{1 / 3}$.

This suggests that we can compare the expansions of various quantities in powers of $\kappa$ between the microscopic and the semiclassical descriptions. For instance, in the 3-brane absorption cross section the expansion parameter is [9]

$N \kappa_{10} \omega^{4} \sim N g_{\text {str }} \alpha^{\prime 2} \omega^{4}$,

where $\omega$ is the incident energy. Thus, we may consider a 'double scaling limit'

$N g_{\mathrm{str}} \rightarrow \infty, \quad \omega^{2} \alpha^{\prime} \rightarrow 0$,

where the expansion parameter (5) is kept small. The semiclassical absorption cross section is naturally expanded in powers of $\omega^{4} \times$ curvature $^{-2}$, which is the same expansion parameter (5) as the one governing the string theoretic description of the 3-branes. The two expansions of the cross-section thus may indeed be compared, and the leading term agrees exactly [9, 10]. This provides strong evidence in favor of absorption by extremal threebranes being a unitary process. While in the classical calculation the information carried by the incident scalar seems to disappear down the infinite throat of the classical solution, the stringy approach indicates that the information is not lost: it is stored in the quantum state of the back-to-back massless gauge bosons on the world volume which are produced by the scalar. Subsequent decay of the threebrane back to the ground state proceeds via annihilation of the gauge bosons into an outgoing massless state, and there seems to be no space for information loss.

$N$ parallel D3-branes are known to be described by a $U(N)$ supersymmetric gauge theory on the world volume [44]. The situation is not as simple in M-theory, where the effective actions of multiple branes are not known in detail. In [9] the scalings of the classical absorption cross-sections 
were found to agree with the twobrane and fivebrane effective action considerations. The absorption cross-section of a longitudinally polarized graviton by a single fivebrane was calculated in 10. From the world-volume effective action it was found that the absorption cross-section is $1 / 4$ of the rate formally predicted by classical gravity. In fact, one could hardly expect perfect agreement for a single fivebrane - the classical description is expected to be valid only for a large number of coincident branes. It is interesting, nevertheless, how close the two calculations come to agreeing with each other. A similar comparison for a single M2-brane reveals a factor discrepancy of $3 \pi /(4 \sqrt{2})$ [10.

\subsection{Semiclassical Absorption by Extremal Branes}

In this section we carry out semiclassical absorption calculations for the three cases of interest: the 3-brane in $D=10$, and the 2- and 5branes in $D=11$. We study the minimally coupled massless scalars whose propagation is governed by

$\nabla^{\mu} \nabla_{\mu} \phi=0$

The extremal 3-brane metric 45] can be written as

$$
\begin{aligned}
d s^{2} & =\left(1+\frac{R^{4}}{r^{4}}\right)^{-1 / 2}\left(-d t^{2}+d x_{1}^{2}+d x_{2}^{2}+d x_{3}^{2}\right) \\
& +\left(1+\frac{R^{4}}{r^{4}}\right)^{1 / 2}\left(d r^{2}+r^{2} d \Omega_{5}^{2}\right) .
\end{aligned}
$$

The s-wave of a minimally coupled massless scalar satisfies

$$
\left[\rho^{-5} \frac{d}{d \rho} \rho^{5} \frac{d}{d \rho}+1+\frac{(\omega R)^{4}}{\rho^{4}}\right] \phi(\rho)=0,
$$

where $\rho=\omega r$. Thus, we are interested in absorption by the Coulomb potential in 6 spatial dimensions. For small $\omega R$ this problem may be solved by matching an approximate solution in the inner region to an approximate solution in the outer region.

To approximate in the inner region, it is convenient to use the variable $z=(\omega R)^{2} / \rho$. Then (9) turns into

$$
\left[\frac{d^{2}}{d z^{2}}-\frac{3}{z} \frac{d}{d z}+1+\frac{(\omega R)^{4}}{z^{4}}\right] \phi=0 .
$$

Substituting $\phi=z^{3 / 2} f(z)$, we find

$$
\left[\frac{d^{2}}{d z^{2}}-\frac{15}{4 z^{2}}+1+\frac{(\omega R)^{4}}{z^{4}}\right] f=0 .
$$

The last term may be ignored if $z \gg(\omega R)^{2}$, i.e. if $\rho \ll 1$. In this region, (11) is easily solved in terms of cylinder functions. Since we are interested in the incoming wave for small $\rho$, the appropriate solution is

$\phi=i z^{2}\left[J_{2}(z)+i N_{2}(z)\right]$,

where $J$ and $N$ are the Bessel and Neumann functions.

Another way to manipulate (9) is by substituting $\phi=\rho^{-5 / 2} \psi$, which gives

$\left[\frac{d^{2}}{d \rho^{2}}-\frac{15}{4 \rho^{2}}+1+\frac{(\omega R)^{4}}{\rho^{4}}\right] \psi=0$.

Now the last term is negligible for $\rho \gg(\omega R)^{2}$, where 13 is solvable in terms of cylinder functions. If $\omega R \ll 1$, then the inner region $(\rho \ll 1)$ overlaps the outer region $\left(\rho \gg(\omega R)^{2}\right)$, and the approximate solutions may be matched. We find that (12) matches onto

$\phi=\frac{32}{\pi} \rho^{-2} J_{2}(\rho), \quad \rho \gg(\omega R)^{2}$.

The absorption probability may be calculated as the ratio of the flux at the throat to the incoming flux at infinity, with the result

$\mathcal{P}=\frac{\pi^{2}}{16^{2}}(\omega R)^{8}$.

In $d$ spatial dimensions, the absorption crosssection is related to the s-wave absorption probability by 46

$$
\sigma=\frac{(2 \pi)^{d-1}}{\omega^{d-1} \Omega_{d-1}} \mathcal{P},
$$

where

$$
\Omega_{D}=\frac{2 \pi^{\frac{D+1}{2}}}{\Gamma\left(\frac{D+1}{2}\right)}
$$


is the volume of a unit $D$-dimensional sphere. Thus, for the 3-brane we find [9]

$\sigma_{3 \text { class }}=\frac{\pi^{4}}{8} \omega^{3} R^{8}$.

The scale parameter $R$ of the classical 3-brane solution is related 40.96 to the number $N$ of coinciding microscopic 3-branes by the equation

$R^{4}=\frac{\kappa_{10}}{2 \pi^{5 / 2}} N$

which follows from the quantization of the threebrane charge. Hence,

$\sigma_{3 \text { class }}=\frac{\kappa_{10}^{2} \omega^{3} N^{2}}{32 \pi}$.

This exercise may be easily repeated for the other two non-dilatonic branes. For the M5-brane the extremal metric is 47

$$
\begin{aligned}
d s^{2} & =\left(1+\frac{R^{3}}{r^{3}}\right)^{-1 / 3}\left(-d t^{2}+d x_{1}^{2}+\ldots+d x_{5}^{2}\right) \\
& +\left(1+\frac{R^{3}}{r^{3}}\right)^{2 / 3}\left(d r^{2}+r^{2} d \Omega_{4}^{2}\right)
\end{aligned}
$$

Now the s-wave problem reduces to absorption by the Coulomb potential in 5 spatial dimensions,

$$
\left[\rho^{-4} \frac{d}{d \rho} \rho^{4} \frac{d}{d \rho}+1+\frac{(\omega R)^{3}}{\rho^{3}}\right] \phi(\rho)=0 .
$$

A matching calculation gives 9]

$\sigma_{5 \text { class }}=\frac{2 \pi^{3}}{3} \omega^{5} R^{9}=\frac{\kappa_{11}^{2} N^{3} \omega^{5}}{2^{6} \cdot 3 \pi^{2}}$,

where $N$ is the number of coincident M5-branes.

For the M2-brane the extremal metric is 48

$$
\begin{aligned}
d s^{2} & =\left(1+\frac{R^{6}}{r^{6}}\right)^{-2 / 3}\left(-d t^{2}+d x_{1}^{2}+d x_{2}^{2}\right) \\
& +\left(1+\frac{R^{6}}{r^{6}}\right)^{1 / 3}\left(d r^{2}+r^{2} d \Omega_{7}^{2}\right)
\end{aligned}
$$

Now the s-wave problem reduces to absorption by the Coulomb potential in 8 spatial dimensions,

$$
\left[\rho^{-7} \frac{d}{d \rho} \rho^{7} \frac{d}{d \rho}+1+\frac{(\omega R)^{6}}{\rho^{6}}\right] \phi(\rho)=0,
$$

${ }^{2}$ By absorption cross section we will consistently mean the cross section per unit longitudinal volume of the brane.
The solution in the inner region is

$$
\phi=i y^{3 / 2}\left[J_{3 / 2}(y)+i N_{3 / 2}(y)\right]
$$

where $y=(\omega R)^{3} /\left(2 \rho^{2}\right)$. This matches onto

$$
\phi=48 \sqrt{\frac{2}{\pi}} \rho^{-3} J_{3}(\rho)
$$

in the outer region. The absorption cross section is found to be 9,49

$$
\sigma_{2 \text { class }}=\frac{\pi^{4}}{12} \omega^{2} R^{9}=\frac{1}{6 \sqrt{2} \pi} \kappa_{11}^{2} \omega^{2} N^{3 / 2} .
$$

\subsection{D-brane approach to absorption}

There is a number of minimally coupled scalars in the theory: the dilaton, the RR scalar, and 'off-diagonal' gravitons polarized with both indices parallel to the 3-brane world-volume [10]. In the semiclassical description they obviously have identical absorption cross-sections. It is remarkable that this is also true in the D-brane description, and that the D-brane absorption crosssection is equal to the semiclassical one at low energies [9, 10].

The threebrane world-volume theory is $\mathcal{N}=4$ supersymmetric $U(N)$ gauge theory where $N$ is the number of parallel threebranes [44]. Thus, the massless fields on the world-volume are the gauge field, 6 scalars, and 4 Majorana fermions, all in the adjoint representation of $U(N)$. The universality of the cross-section is not trivial in the world-volume description: while for dilatons and RR scalars leading absorption proceeds by conversion into a pair of gauge bosons only, for the gravitons polarized along the brane it involves a summation over conversions into world-volume scalars, fermions, and gauge bosons [10].

The world-volume action, excluding all couplings to external fields, is $(I=1, \ldots, 4 ; i=$ $4, \ldots, 9)$

$$
\begin{gathered}
S_{3}=T_{(3)} \int d^{4} x \operatorname{Tr}\left[-\frac{1}{4} F_{\alpha \beta}^{2}+\frac{i}{2} \bar{\psi}^{I} \gamma^{\alpha} \partial_{\alpha} \psi_{I}\right. \\
\left.-\frac{1}{2}\left(\partial_{\alpha} X^{i}\right)^{2}+\text { interactions }\right] .
\end{gathered}
$$

We also need to know how the threebrane worldvolume fields couple to the bulk fields of type IIB 
supergravity. For the terms necessary to us, the generalization from $U(1)$ to $U(N)$ gauge group is straightforward. One can, in principle, obtain detailed information about the structure of the non-abelian action by directly computing string amplitudes as done in [8].

The leading bosonic terms in the action for a single 3-brane in a type IIB supergravity background are [3,50]

$$
\begin{aligned}
S_{3} & =-T_{(3)} \int d^{4} x\left(\sqrt{-\operatorname{det}\left(\hat{g}+e^{-\phi / 2} \mathcal{F}\right)}\right. \\
& \left.+\frac{1}{4 !} \varepsilon^{\alpha \beta \sigma \rho} \hat{C}_{\alpha \beta \sigma \rho}+\frac{1}{2} \hat{C}_{\alpha \beta} \tilde{\mathcal{F}}^{\alpha \beta}+\frac{1}{4} C \mathcal{F}_{\alpha \beta} \tilde{\mathcal{F}}^{\alpha \beta}\right)
\end{aligned}
$$

where

$$
\begin{gathered}
\mathcal{F}_{\alpha \beta}=F_{\alpha \beta}+\hat{B}_{\alpha \beta}, \\
\tilde{F}^{\alpha \beta}=\frac{1}{2} \varepsilon^{\alpha \beta \sigma \rho} F_{\sigma \rho}, \\
\hat{g}_{\alpha \beta}=g_{M N} \partial_{\alpha} X^{M} \partial_{\beta} X^{N}, \text { etc. },
\end{gathered}
$$

and the background fields are functions of $X^{M}$. In the static gauge $\left(X^{\alpha}=x^{\alpha}, \alpha=0, \ldots, 3\right)$ one has

$$
\hat{g}_{\alpha \beta}=g_{\alpha \beta}+2 g_{i(\alpha} \partial_{\beta)} X^{i}+g_{i j} \partial_{\alpha} X^{i} \partial_{\beta} X^{j} .
$$

The additional fermionic terms are dictated by supersymmetry.

The leading-order interaction of the dilaton with world-volume fields implied by (24) was first discussed in [9]. The coupling of the RR scalar $C$ is similar, being related by $S L(2, R)$ duality. We also include the coupling of the gravitons polarized parallel to the brane, $h_{\alpha \beta}=g_{\alpha \beta}-\eta_{\alpha \beta}$.

Generalizing to $U(N)$, we find that the part of $S_{\text {int }}$ that is relevant to the leading-order absorption processes we wish to consider is

$$
\begin{aligned}
S_{\mathrm{int}}=T_{(3)} \int d^{4} x & {\left[\operatorname{Tr}\left(\frac{1}{4} \phi F_{\alpha \beta}^{2}-\frac{1}{4} C F_{\alpha \beta} \tilde{F}^{\alpha \beta}\right)\right.} \\
+ & \left.\frac{1}{2} h^{\alpha \beta} T_{\alpha \beta}\right],
\end{aligned}
$$

where

$$
\begin{aligned}
T_{\alpha \beta}= & \operatorname{Tr}\left[F_{\alpha}^{\gamma} F_{\beta \gamma}-\frac{1}{4} \eta_{\alpha \beta} F_{\gamma \delta}^{2}-\frac{i}{2} \bar{\psi}^{I} \gamma_{(\alpha} \partial_{\beta)} \psi_{I}\right. \\
& \left.+\partial_{\alpha} X^{i} \partial_{\beta} X^{i}-\frac{1}{2} \eta_{\alpha \beta}\left(\partial_{\gamma} X^{i}\right)^{2}\right] .
\end{aligned}
$$

${ }^{3}$ We ignore the fermionic couplings like $\phi \bar{\psi}^{I} \gamma^{\alpha} \partial_{\alpha} \psi_{I}$ and similar ones for $C$ and $h_{\alpha \beta}$ which are proportional to the fermionic equations of motion and thus give vanishing contribution to the S-matrix elements.
Let us first consider an off-diagonal graviton polarized along the brane, say $h_{x y}$. From (24) one can read off the invariant amplitudes for absorption into two scalars, two fermions, or two gauge bosons (we have to include $\sqrt{2} \kappa_{10}$, the field normalization factor for $h_{x y}$ ). Summing over different species and polarizations of particles available (six different $X^{i}$, for example), one obtains

$$
\begin{aligned}
\text { scalars } & : \overline{\mid \mathcal{M}}^{2}=3 \kappa_{10}^{2} \omega^{4} n_{x}^{2} n_{y}^{2} \\
\text { fermions } & : \overline{|\mathcal{M}|}=\kappa_{10}^{2} \omega^{4}\left(n_{x}^{2}+n_{y}^{2}-4 n_{x}^{2} n_{y}^{2}\right) \\
\text { vectors } & : \quad \overline{\mathcal{M}}^{2}=\kappa_{10}^{2} \omega^{4}\left(1-n_{x}^{2}-n_{y}^{2}+n_{x}^{2} n_{y}^{2}\right)
\end{aligned}
$$

where $\vec{n}$ is the direction of one of the outgoing particles. We have anticipated conservation of energy and momentum by setting $\vec{p}_{1}+\vec{p}_{2}=0$ and $\omega_{1}+\omega_{2}=\omega$. It is remarkable that the sum of these three quantities is independent of $\vec{n}$. Thus, if one sums over all the states in the $\mathcal{N}=4$ supermultiplet, the result is isotropic:

$\overline{\mid \mathcal{M}}^{2}=\kappa_{10}^{2} \omega^{4}$.

The absorption cross-section is evaluated from $\overline{\mid \mathcal{M}}^{2}$ in precisely the same way that decay rates of massive particles are calculated in conventional 4-dimensional field theories:

$$
\begin{gathered}
\sigma_{3 \mathrm{abs}}=\frac{N^{2}}{2} \frac{1}{2 \omega} \int \frac{d^{3} p_{1}}{(2 \pi)^{3} 2 \omega_{1}} \frac{d^{3} p_{2}}{(2 \pi)^{3} 2 \omega_{2}} \\
(2 \pi)^{4} \delta^{4}\left(q-\sum_{i} p_{i}\right) \overline{|\mathcal{M}|^{2}} .
\end{gathered}
$$

The leading factor of $N^{2}$ accounts for the multiple branes and the $1 / 2$ is present because the outgoing particles are identical. The cross-section agrees with the semi-classical $\ell=0$ result, (18),

$\sigma_{3 \text { abs }}=\frac{\kappa_{10}^{2} \omega^{3} N^{2}}{32 \pi}=\sigma_{3 \text { class. . }}$

The D-brane calculations for the dilaton and the RR scalar yield identical results, in agreement with the semiclassical gravity 9,10$]$.

It is important to examine the structure of higher power in $g_{\text {str }}$ corrections to the crosssection [51]. In semiclassical supergravity the only quantity present is $\kappa$, and corrections to (18) can only be of the form

$a_{1} \kappa^{3} \omega^{7}+a_{2} \kappa^{4} \omega^{11}+\ldots$ 
However, in string theory we could in principle find corrections even to the leading term $\sim \omega^{3}$, so that

$$
\begin{gathered}
\sigma_{3 \text { abs }}=\frac{\kappa^{2} \omega^{3} N^{2}}{32 \pi}\left(1+b_{1} g_{\mathrm{str}} N+b_{2}\left(g_{\mathrm{str}} N\right)^{2}+\ldots\right) \\
+\mathcal{O}\left(\kappa^{3} \omega^{7}\right) .
\end{gathered}
$$

Presence of such corrections would spell a manifest disagreement with supergravity because, as we have explained, the comparison has to be carried out in the limit $N g_{\text {str }} \rightarrow \infty$. Luckily, $b_{i}=0$ due to certain non-renormalization theorems in $D=4 \mathcal{N}=4$ SYM theory. In [52] a detailed argument was presented for the absence of such corrections in the absorption cross-section of gravitons polarized along the brane. These gravitons couple to the stress-energy tensor on the world volume, and it was shown that the absorption cross-section is determined by the central term in its two-point function. Schematically, one finds

$T(x) T(0)=\frac{c}{x^{8}}+\ldots$.

The fact that $b_{i}=0$ follows from the fact that the one-loop calculation of the central charge is exact in $D=4 \mathcal{N}=4$ SYM theory, yielding $c=N^{2} / 4$ $53,54,52$.

A more difficult comparison is the absorption cross-section for higher partial waves. In [9] the following term was proposed to describe absorption of a dilaton in the $\ell$-th partial wave,

$T_{(3)} \int d^{4} x \frac{1}{4 \cdot \ell !} \partial_{i_{1}} \cdots \partial_{i_{\ell}} \phi \operatorname{Tr}\left(X^{i_{1}} \cdots X^{i_{\ell}} F_{\alpha \beta} F^{\alpha \beta}\right)$

This term originates from Taylor expansion of the background field $\phi(X)$ in the world volume action: it describes a process where an incident dilaton is converted into $\ell+2$ massless $D$-brane excitations. It was shown in 9 that this term predicts a cross-section for the $\ell$-th partial wave whose scaling with $\omega$ and $N$ is in agreement with classical gravity. In 10] precise agreement in the normalizations was found for $\ell=0,1$, but for $\ell>1$ the D-brane cross-section was found to be bigger than the semiclassical one. Some suggestion for how to restore agreement were given in 10, but the puzzle has not been resolved. This is an interesting problem for the future.

\subsection{Comparing absorption cross-sections for M-branes}

A remarkable aspect of the agreement between the string theoretic and the classical results for threebranes is that it holds exactly for any value of $N$, including $N=1$. As explained in the introduction, the classical geometry should be trusted only in the limit $g_{\text {str }} N \rightarrow \infty$. Thus, the agreement of the absorption cross-sections for $N=1$ suggests that our calculations are valid even in the limit $g_{\mathrm{str}} \rightarrow \infty$, provided that $g_{\mathrm{str}} \alpha^{\prime 2} \omega^{4}$ is kept small. The supersymmetric non-renormalization theorems are probably at work here, insuring that there are no string loop corrections. We would like to ask whether the exact agreement between the absorption cross-sections is also found for the twobranes and fivebranes of M-theory. Their effective actions are not known for $N>1$, so we can compare the cross-sections only for $N=1$. In contrast to the threebranes, there is no exact agreement in the normalizations [10], which is probably due to the fact that M-theory has no parameter like $g_{\text {str }}$ that can be dialed to make the classical solution reliable. We will now review some of the calculations in [10].

First we discuss absorption of longitudinally polarized gravitons by a twobrane. The massless fields in the effective action are 8 scalars and 8 Majorana fermions. The longitudinal graviton couples to the energy momentum tensor on the world-volume, $T_{\alpha \beta}$. The terms in the effective action necessary to describe the absorption of $h_{x y}$ are $(i=3, \ldots, 10 ; I=1, \ldots, 8)$

$$
\begin{gathered}
T_{(2)} \int d^{3} x\left[-\frac{1}{2} \partial_{\alpha} X^{i} \partial^{\alpha} X^{i}+\frac{i}{2} \bar{\psi}^{I} \gamma^{\alpha} \partial_{\alpha} \psi^{I}\right. \\
\left.+\sqrt{2} \kappa_{11} h_{x y}\left(\partial_{x} X^{i} \partial_{y} X^{i}-\frac{i}{4} \bar{\psi}^{I}\left(\gamma_{x} \partial_{y}+\gamma_{y} \partial_{x}\right) \psi^{I}\right)\right],
\end{gathered}
$$

where $h_{x y}$ is the canonically normalized field which enters the $D=11$ space-time action as

$$
-\frac{1}{2} \int d^{11} x \partial_{M} h_{x y} \partial^{M} h_{x y} .
$$

The absorption cross-section is found using the Feynman rules in a way analogous to the threebrane calculation. For the 8 scalars, we find that the matrix element squared (with all the relevant 
factors included) is

$$
\frac{\kappa_{11}^{2} \omega^{4}}{2} 4 n_{x}^{2} n_{y}^{2}
$$

where $\vec{n}$ is the unit vector in the direction of one of the outgoing particles. For the 8 Majorana fermions, the corresponding object summed over the final polarizations is

$$
\frac{\kappa_{11}^{2} \omega^{4}}{2}\left(n_{x}^{2}-n_{y}^{2}\right)^{2} .
$$

Adding them up, we find that the dependence on direction cancels out, just as in the threebrane case. The sum must be multiplied by the phase space factor $\frac{1}{8 \omega^{2}}$, so that the total cross-section is $\sigma_{2 \mathrm{abs}}=\frac{\kappa_{11}^{2} \omega^{2}}{16}$.

This does not agree with the classical result (22) for $N$ set to 1 . Notice that even the power of $\pi$ does not match. This situation is reminiscent of the discrepancy in the near-extremal entropy where the relative factor was a transcendental number involving $\zeta(3) 41$.

Now we turn to the fivebrane. The massless fields on the fivebrane form a tensor multiplet consisting of 5 scalars, 2 Weyl fermions and the antisymmetric tensor $\mathcal{B}_{\alpha \beta}$ with anti-selfdual strength. To discuss the absorption of longitudinally polarized gravitons, $h_{x y}$, we need the action $(i=6, \ldots, 10 ; I=1,2)$

$$
\begin{aligned}
& T_{(5)} \int d^{6} x\left[-\frac{1}{2} \partial_{\alpha} X^{i} \partial^{\alpha} X^{i}-\frac{1}{12} \mathcal{H}_{\alpha \beta \gamma}^{2}+i \bar{\psi}^{I} \gamma^{\alpha} \partial_{\alpha} \psi^{I}\right. \\
& +\quad \sqrt{2} \kappa_{11} h_{x y}\left(\partial_{x} X^{i} \partial_{y} X^{i}+\frac{1}{2} \mathcal{H}_{x \beta \gamma}^{-} \mathcal{H}_{y}^{-\beta \gamma}\right. \\
& \left.\left.\quad-\quad \frac{i}{2} \bar{\psi}^{I}\left(\gamma_{x} \partial_{y}+\gamma_{y} \partial_{x}\right) \psi^{I}\right)\right]
\end{aligned}
$$

For the 5 scalars, we find that the matrix element squared (with all the relevant factors included) is

$\frac{\kappa_{11}^{2} \omega^{4}}{2} \frac{5}{2} n_{x}^{2} n_{y}^{2}$

For the 2 Weyl fermions, the corresponding object summed over the final polarizations is

$\frac{\kappa_{11}^{2} \omega^{4}}{2}\left(n_{x}^{2}+n_{y}^{2}-4 n_{x}^{2} n_{y}^{2}\right)$.
Finally, the contribution of the anti-selfdual gauge field turns out to be equal to that of the usual, unconstrained $\mathcal{H}_{\alpha \beta \gamma}$ divided by 2. Summing over polarizations, we find,

$\frac{\kappa_{11}^{2} \omega^{4}}{2}\left(1-n_{x}^{2}-n_{y}^{2}+\frac{3}{2} n_{x}^{2} n_{y}^{2}\right)$.

Adding up the contributions of the entire tensor multiplet, we find that all the directiondependent terms cancel out, just as they did for the threebrane and the twobrane. Multiplying by the phase space factor $\frac{\omega}{2^{7} \cdot 3 \pi^{2}}$, we find that the total cross-section for the longitudinally polarized gravitons is

$\sigma_{5 \text { abs }}=\frac{\kappa_{11}^{2} \omega^{5}}{2^{8} \cdot 3 \pi^{2}}$.

This turns out to be a factor of 4 smaller than the classical result (20) evaluated for $N=1$.

What is the reason for this discrepancy? For $N=1$, the curvature of the solution is of order of the 11-dimensional Planck scale. Obviously, the 11-dimensional supergravity is at best a low-energy approximation to M-theory. The M-theory effective action should contain higherderivative terms weighted by powers of $\kappa_{11}$, by analogy with the $\alpha^{\prime}$ and $g_{\text {str }}$ expansions of the string effective action. Thus, for $N=1$, the classical solution may undergo corrections of order one which we believe to be the source of the discrepancy. For large $N$, however, we expect the M-theory cross-section to agree exactly with the classical cross-section. On the other hand, the absorption cross-section for gravitons polarized along the brane is in general related to the Schwinger term in the two-point function of the stress-energy tensors [52]. The form of the semiclassical cross-section for $N$ M2-branes (22) indicates that we are dealing with a superconformal field theory in $2+1$ dimensions. Schematically, the OPE is

$T(x) T(0)=\frac{c_{2}}{x^{6}}+\ldots$,

and the behavior of the central charge is $c_{2} \sim$ $N^{3 / 2}$ for large $N$ 52]. For $N$ M5-branes the semiclassical cross-section (20) indicates that we are dealing with a superconformal field theory in $5+1$ 
dimensions. Now the schematic OPE of stressenergy tensors is

$T(x) T(0)=\frac{c_{5}}{x^{12}}+\ldots$,

and the behavior of the central charge is $c_{5} \sim N^{3}$ for large $N$ [52].

These results have an obvious connection with properties of the near-extremal entropy found in 411. Indeed, the near-extremal entropy of a large number $N$ of coincident M2-branes is formally reproduced by $\mathcal{O}\left(N^{3 / 2}\right)$ massless free fields in $2+1$ dimensions, while that of $N$ coincident M5-branes is reproduced by $\mathcal{O}\left(N^{3}\right)$ massless free fields in $5+1$ dimensions.

\section{Creation of Fundamental Strings by Crossing D-branes}

The D-branes are BPS saturated objects which preserve 16 supersymmetries out of the original 32. This implies that when two Dirichlet $p$-branes are placed parallel to each other, the force between them vanishes. A string theoretic calculation of this force involves the cylinder diagram, with the ends of the cylinder attached to different D-branes. In the open string channel there are contributions from the NS, R, and NS $(-1)^{F}$ sectors, which cancel due to the abstruse identity for theta-functions [2]. Physically, this means that the attraction due to NS-NS closed strings, the graviton and the dilaton, is canceled by the repulsion of the like $\mathrm{R}-\mathrm{R}$ charges.

It is interesting to study a more general situation where a $p^{\prime}$-brane is placed parallel to a $p$-brane with $p^{\prime}<p$. This configuration preserves 8 of the supersymmetries if $p-p^{\prime}=4$ or 8 [3]. For $p-p^{\prime}=4$ only the NS and the R open string sectors contribute to the cylinder amplitude, and they cancel identically. Thus, there is no force due to the R-R exchange, while the graviton and the dilaton forces cancel identically.

A more complicated situation arises for $p-p^{\prime}=$ 8. One example of this is the 0 -brane near the 8 brane, which is important for understanding the heterotic theory 55. Now the contribution of the NS and R open string sectors to the cylinder amplitude is [56

$$
\begin{aligned}
& A_{\mathrm{NS}-\mathrm{NS}}=\frac{1}{2} \int_{0}^{\infty} \frac{d t}{t}\left(8 \pi^{2} \alpha^{\prime} t\right)^{-1 / 2} e^{-\frac{t Y^{2}}{2 \pi \alpha^{\prime}}} f_{4}^{-8}(q) \times \\
& \left(-f_{2}^{8}(q)+f_{3}^{8}(q)\right)=\frac{1}{2} \int_{0}^{\infty} \frac{d t}{t}\left(8 \pi^{2} \alpha^{\prime} t\right)^{-1 / 2} e^{-\frac{t Y^{2}}{2 \pi \alpha^{\prime}}}
\end{aligned}
$$

where $q=e^{-\pi t}$ and $Y$ is the transverse position of the 0-brane relative to the 8-brane. Thus, we find a constant repulsive force due to the NS-NS closed strings,

$$
-\frac{\partial A_{\mathrm{NS}-\mathrm{NS}}(Y)}{\partial Y}=\frac{1}{4 \pi \alpha^{\prime}} \operatorname{sign}(Y) \text {. }
$$

As pointed out by Lifschytz [56], this is canceled by a contribution of the $\mathrm{R}(-1)^{F}$ open string sector, which implies that there is attraction due to $\mathrm{R}-\mathrm{R}$ exchange. The nature of this attraction was elucidated in [11].

A peculiar feature of this force is that it jumps by $\pm \frac{1}{2 \pi \alpha^{\prime}}$ every time the 0 -brane crosses the 8brane. This jump is due to creation of a fundamental string stretched between the 0-brane and the 8-brane [11. This phenomenon is similar, and in fact U-dual, to the creation of a 3-brane discovered by Hanany and Witten 12. Since the number of stretched fundamental strings jumps by \pm 1 upon each crossing, we may regard the ground state of the $0-8$ system as containing $\pm \frac{1}{2}$ of a fundamental string (the sign refers to whether the string enters or exits the 0-brane). When the 0-brane is to the left of the 8-brane, we have, say, $-\frac{1}{2}$ of a fundamental string. Upon crossing, this turns into $+\frac{1}{2}$. The attractive force equal to $\frac{1}{2}$ of the fundamental string tension is what is necessary to cancel the repulsion due to the graviton and the dilaton, (41). This is how the no-force condition required by supersymmetry is maintained in the 0-8 system.

\subsection{U-duality and creation of a fundamen- tal string}

The creation of a stretched string by a 0-brane crossing an 8-brane is related by U-duality to creation of a stretched 3-brane by a R-R 5-brane crossing a NS-NS 5-brane. Hanany and Witten showed that, when an R-R charged 5-brane positioned in the $(1-2-6-7-8)$ directions crosses a NS-NS charged 5-brane positioned in the $(1-2-3-4-5)$ directions, a single $(1-2-9)$ 
3 -brane stretched between the 5 -branes is created 12.

Applying T-duality along directions 1 and 2 we find that, when a $(6-7-8) 3$-brane crosses a ( $1-2-3-4-5)$ NS-NS 5 -brane, then a D-string stretched between them along the 9 th direction is created. From the S-duality of the type IIB theory it now follows that, when a $(6-7-8) 3$-brane crosses a $(1-2-3-4-5)$ R-R 5 -brane, then a fundamental string stretched between them along the 9 th direction is created. This is the kind of process that is of primary interest to us, because it involves two D-branes with $8 \mathrm{ND}$ coordinates. There are a number of other such processes related to this by T-duality. For example, after Tdualizing along directions 6,7 and 8 , we find that a 0 -brane crossing an 8-brane creates a stretched fundamental string.

It is interesting that both the Hanany-Witten process and the fundamental string creation originate from the same phenomenon in M-theory: creation of a 2-brane by crossing 5 -branes. Indeed, when a $(2-3-4-5-10) 5$-brane crosses a $(6-7-8-9-10) 5$-brane, a $(1-10) 2$-brane stretched between the 5-branes is created. Reducing to the type IIA theory along direction 5, we find that a 4-brane crossing a 5 -brane creates a 2-brane. This is T-dual to the 3 -brane creation discussed in 12]. We may, however, choose to reduce to the type IIA theory along direction 10 , which is common to all the branes. Then we find that a $(2-3-4-5)$ 4-brane crossing a $(6-7-8-9)$ 4-brane creates a fundamental string stretched along direction 1. This confirms that two crossing D-branes, positioned in such a way that there are $8 \mathrm{ND}$ coordinates, create a stretched fundamental string.

\subsection{Effective action arguments}

Let us give a direct argument for the creation of fundamental strings. For concreteness, we will refer to the 0-8 system, but analogous arguments apply to all cases related to this by T-duality.

The term in the 8-brane world volume action which is crucial for our purposes is 2 ]

$\mu_{(8)} \frac{1}{2 \cdot 7 !} \int d^{9} \sigma \varepsilon_{\nu_{0} \ldots \nu_{8}} C_{(7)}^{\nu_{0} \ldots \nu_{6}} F^{\nu_{7} \nu_{8}}$ where $C_{(7)}$ is an R-R potential, and $F=d A$ is the world volume gauge field strength. The D-brane charge densities were determined in [2] to be

$$
\mu_{(p)}=\sqrt{2 \pi}\left(2 \pi \sqrt{\alpha^{\prime}}\right)^{3-p} .
$$

Integrating (42) by parts, we get

$$
\begin{gathered}
\frac{\mu_{(8)}}{8 !} \int d^{9} \sigma \varepsilon_{\nu_{0} \ldots \nu_{8}} F_{(8)}^{\nu_{0} \ldots \nu_{7}} A^{\nu_{8}} \\
=\mu_{(8)} \int d^{9} \sigma F_{(2)}^{\mu 9} A_{\mu},
\end{gathered}
$$

where

$$
F_{(8)}=d C_{(7)}, \quad F_{(2)}={ }^{*} F_{(8)},
$$

and 9 is the direction normal to the 8-brane.

In the presence of a stationary 0-brane, there is a radial electric field,

$F_{(2)}^{0 r}=\frac{\mu_{(0)}}{r^{8} \Omega_{8}}$,

where $\Omega_{8}$ is the volume of a unit 8-sphere. Eq. (43) shows that the normal component of the electric field, $F_{(2)}^{09}$, plays the role of the charge density in the world volume gauge theory. The total charge on the 8-brane is

$\mu_{(8)} \int d^{8} \sigma F_{(2)}^{09}=\frac{1}{2} \mu_{(8)} \mu_{(0)}=\frac{1}{4 \pi \alpha^{\prime}}$.

Let us recall that an endpoint of a fundamental string manifests itself in the world volume gauge theory as an electric charge of magnitude $\pm \frac{1}{2 \pi \alpha^{\prime}}$. We conclude that the 0-brane and the 8-brane are connected by one half of a fundamental string. This provides the attraction that cancels the repulsion from the graviton-dilaton exchange.

As the 0-brane crosses the 8-brane, the net electric charge on the 8 -brane jumps from $\frac{1}{4 \pi \alpha^{\prime}}$ to $-\frac{1}{4 \pi \alpha^{\prime}}$. This clearly shows that an endpoint of a fundamental string is created on the 8-brane. Similar considerations in the 0-brane action show that the other end of the string is attached to the 0 -brane. The term in the 0 -brane action responsible for this effect is

$\mu_{(0)} \int d \tau F A_{0}$,

where $F={ }^{*} F_{(10)}$ is the zero-form field strength dual to the 10 -form emitted by the 8 -brane. Thus, 
$\mu_{(0)} F$ is the 'source' for $A_{0}$. We believe that this shows that the fundamental string indeed ends on the 0-brane. . $^{2}$ Correctness of this argument may be checked through T-duality. For instance, if we T-dualize the $0-8$ system to a pair of orthogonal 4 -branes, then (46) goes into the following term of the 4-brane action,

$$
\frac{\mu_{(4)}}{4 !} \int d^{5} \sigma \varepsilon_{\nu_{0} \ldots \nu_{4}} F_{(4)}^{\nu_{0} \nu_{1} \nu_{2} \nu_{3}} A^{\nu_{4}} \text {. }
$$

The jump in the total charge on a 4-brane as it is crossed by the other 4-brane is

$$
\mu_{(4)}^{2}=\frac{1}{2 \pi \alpha^{\prime}}
$$

which is precisely the tension of one fundamental string.

The 'bare' Chern-Simons term (46) is crucial for the consistency of the quantum mechanics of a 0 -brane near a 8-brane. The 0-8 strings are described by a complex fermion $\chi$ with the lagrangian

$$
L_{\chi}=-i \bar{\chi} \dot{\chi}-\bar{\chi} Y \chi-\bar{\chi} A_{0} \chi
$$

where the scalar $Y$ is the distance between the 0 -brane and the 8 -brane (we are now working in the units where $2 \pi \alpha^{\prime}=1$ ).

If we integrate out the fermions, we find 'induced' Chern-Simons and potential terms 60]

$\frac{1}{2} \operatorname{sign}(Y)\left(Y+A_{0}\right)$.

Thus, the theory seems anomalous because the coefficient in front of the Chern-Simons term is fractional. This problem is solved by the creation of the fundamental strings. The 'bare' ChernSimons term (46),

$$
\mu_{(0)} F A_{0}=-\frac{1}{2} \operatorname{sign}(Y) A_{0},
$$

\footnotetext{
4 A similar conclusion was reached in 57,58 . In the presence of an 8-brane the type IIA supergravity is massive, and the equations of motion imply that a string must end on a 0-brane. A careful normalization shows that, when the 0 -brane is to the right (left) of the 8 -brane, $-\frac{1}{2}\left(+\frac{1}{2}\right)$ of a fundamental string ends on the 0-brane. Brane creation has also been analyzed using the anomaly inflow argument [59].
}

exactly cancels the 'induced' term in (48). The 'bare' term may be interpreted as due to $1 / 2$ of a fundamental string. There is a corresponding 'bare' attractive potential related to (46) by $N=$ 8 supersymmetry [60],

$$
\delta L=-\frac{1}{2}|Y|,
$$

which cancels the 'induced' repulsive potential. Thus, the entire 'induced' lagrangian (48) obtained by integrating over the fermions is canceled by the 'bare' terms due to $1 / 2$ of a fundamental string.

In the previous section we showed that the string creation follows by dimensional reduction from membrane creation in M-theory. Let us make a direct argument for the latter. Consider the effective action for a $(1-2-3-4-5) 5$-brane in the presence of a $(1-6-7-8-9) 5$-brane. This action contains a Chern-Simons term

$q_{(5)} \frac{1}{(3 !)^{2}} \int d^{6} \sigma \varepsilon_{\nu_{0} \ldots \nu_{5}} C^{\nu_{0} \nu_{1} \nu_{2}} H^{\nu_{3} \nu_{4} \nu_{5}}$,

where $H=d B$ is the world volume field strength. The 2-brane and 5 -brane charge densities and tensions were normalized in [19],

$$
\begin{aligned}
& q_{(2)}=\sqrt{2} \kappa T_{(2)}=\sqrt{2}\left(2 \kappa \pi^{2}\right)^{1 / 3}, \\
& q_{(5)}=\sqrt{2} \kappa T_{(5)}=\sqrt{2}\left(\frac{\pi}{2 \kappa}\right)^{1 / 3} .
\end{aligned}
$$

Integrating (49) by parts, we find

$q_{(5)} \frac{1}{2 \cdot 4 !} \int d^{6} \sigma \varepsilon_{\nu_{0} \ldots \nu_{5}} F^{\nu_{0} \nu_{1} \nu_{2} \nu_{3}} B^{\nu_{4} \nu_{5}}$.

This shows that $F_{2345}$ acts as a source for $B_{01}$. Thus, $F_{2345}$ is proportional to the density of strings on the world volume which point along direction 1 . Such a string is the boundary of a $(1-10)$ 2-brane stretched between the 5 -branes. Evaluating the flux through the $(1-2-3-4-5)$ 5 -brane due to the $(1-6-7-8-9) 5$-brane,

$$
\int d^{5} \sigma F_{2345}
$$

we find that the net charge that couples to $B_{01}$ is $\frac{q_{(5)}^{2}}{2}=\frac{T_{(2)}}{2}$. 
Thus, one half of a 2-brane is stretched between the 5-branes. As the 5-branes pass through each other, one 2-brane is created. It is interesting that this process is encoded in the relation (53) between the charge of the 5 -brane and the tension of the 2-brane in M-theory.

\subsection{Implications for the type I' theory}

The phenomena we have discussed have interesting implications for the physics of the type I' theory, which is S-dual to the heterotic string. Its set-up involves 16 D8-branes located between two 8 -orientifold planes. The dynamics of the zerobranes is described by the quantum mechanical system introduced in [55]. At first sight it appears that there exists an effective linear potential for the 0-brane which experiences a discontinuity in the slope every time a 0-brane passes through an 8-brane. This potential, however, results from including only the NS-NS exchange in the string theory cylinder diagram. As we have shown, inclusion of the R-R term interpretable as the string creation cancels the discontinuity in the force. In fact, the entire linear potential cancels everywhere [1]. Similarly the net Chern-Simons term also cancels, as required by the $N=8$ supersymmetry. This is consistent with the picture that we have developed in the preceding sections.

\section{Acknowledgments}

These notes are based on conference talks delivered at Strings '97 in Amsterdam and at SUSY '97 in Philadelphia. I thank the organizers of these conferences for their hospitality. I am grateful to U. Danielsson, G. Ferretti, S. Gubser and A. Tseytlin for collaboration on the material presented here. This work was supported in part by the DOE grant DE-FG0291ER40671, the NSF Presidential Young Investigator Award PHY-9157482, and the James S. McDonnell Foundation grant No. 91-48.

\section{REFERENCES}

1. J. Dai, R. G. Leigh, and J. Polchinski, Mod. Phys. Lett. A4, 2073 (1989);

P. Horava, Nucl. Phys. B327 (1989) 461, Phys. Lett. B231 (1989) 251;
G. Pradisi and A. Sagnotti, Phys. Lett. B216 (1989) 59;

A. Sagnotti, Phys. Rept. 184 (1989) 167;

R. G. Leigh, Mod. Phys. Lett. A4, 2767 (1989);

M.B. Green, Phys. Lett. B266 (1991) 325.

2. J. Polchinski, Phys. Rev. Lett. 75, 4724 (1995).

3. J. Polchinski, S. Chaudhuri, and C. V. Johnson, "Notes on D-Branes," hep-th/9602052. J. Polchinski, "TASI Lectures on D-branes," hep-th/9611050.

4. For a review, see A. Hashimoto and I.R. Klebanov, Nucl. Phys. Proc. Suppl. 55B (1997) 118, hep-th/9611214.

5. I. R. Klebanov and L. Thorlacius, Phys. Lett. B371 (1996) 51-56.

6. S.S. Gubser, A. Hashimoto, I.R. Klebanov, and J.M. Maldacena, Nucl. Phys. 472 (1996) 231-248, hep-th/9601057.

7. M. R. Garousi and R. C. Myers, "Superstring Scattering from D-Branes," Nucl. Phys. 475 (1996) 193-224, hep-th/9603194.

8. A. Hashimoto and I.R. Klebanov, "Decay of Excited D-branes," Phys. Lett. B381 (1996) 437-445, hep-th/9604065.

9. I.R. Klebanov, Nucl. Phys. 496 (1997) 231, hep-th/9702076.

10. S.S. Gubser, I.R. Klebanov and A.A. Tseytlin, Nucl. Phys. B499 (1997) 217, hepth/9703040.

11. U. Danielsson, G. Ferretti and I.R. Klebanov, Phys. Rev. Lett. 79 (1997) 1984, hepth/9705084.

12. A. Hanany and E. Witten, Nucl. Phys. B492 (1997) 152, hep-th/9611230.

13. M. Cvetič and A.A. Tseytlin, Phys. Lett. B366 (1996) 95, hep-th/9510097; Phys. Rev. D53 (1996) 5619, hep-th/9512031.

14. A. Strominger and C. Vafa, Phys. Lett. B379 (1996) 99, hep-th/9601029.

15. C.G. Callan and J.M. Maldacena, Nucl. Phys. B472 (1996) 591, hep-th/9602043.

16. J.M. Maldacena and A. Strominger, Phys. Rev. Lett. 77 (1996) 428, hep-th/9603060.

17. C. Johnson, R. Khuri and R. Myers, Phys. Lett. B378 (1996) 78, hep-th/9603061.

18. A.A. Tseytlin, Nucl. Phys. B475 (1996) 49, 
hep-th/9604035.

19. I.R. Klebanov and A.A. Tseytlin, Nucl. Phys. B475 (1996) 179, hep-th/9604166.

20. V. Balasubramanian and F. Larsen, Nucl. Phys. B478 (1996) 199, hep-th/9604189.

21. J.M. Maldacena and L. Susskind, Nucl. Phys. B475 (1996) 679, hep-th/9604042.

22. A. Dhar, G. Mandal and S. R. Wadia, Phys. Lett. B388 (1996) 51, hep-th/9605234.

23. S.R. Das and S.D. Mathur, Nucl. Phys. B478 (1996) 561, hep-th/9606185.

24. S.S. Gubser and I.R. Klebanov, Nucl. Phys. B482 (1996) 173, hep-th/9608108.

25. J.M. Maldacena and A. Strominger, Phys. Rev. D55 (1997) 861, hep-th/9609026.

26. S.S. Gubser and I.R. Klebanov, Phys. Rev. Lett. 77 (1996) 4491, hep-th/9609076.

27. C.G. Callan, Jr., S.S. Gubser, I.R. Klebanov and A.A. Tseytlin, Nucl. Phys. B489 (1997) 65, hep-th/9610172; I.R. Klebanov and M. Krasnitz, Phys. Rev. D55 (1997) 3250, hepth/9612051.

28. I.R. Klebanov and S. Mathur, Nucl. Phys. B500 (1997) 115, hep-th/9701187.

29. S. Hawking and M. Taylor-Robinson, hepth/9702045.

30. F. Dowker, D. Kastor and J. Traschen, hepth/9702109.

31. M. Krasnitz and I.R. Klebanov, Phys. Rev. D56 (1997) 2173, hep-th/9703216.

32. I.R. Klebanov, A. Rajaraman and A. Tseytlin, hep-th/9704112.

33. S. Mathur, hep-th/9704156.

34. S. Gubser, hep-th/9704195.

35. M. Cvetič and F. Larsen, hep-th/9705192, hep-th/9706071.

36. S. Ferrara and R. Kallosh, hep-th/9602136, hep-th/9603090; B. Kol and A. Rajaraman, hep-th/9608126.

37. T. Banks, W. Fischler, S. Shenker and L. Susskind, Phys. Rev. D55 (1997) 5112, hepth/9610043.

38. T. Banks, W. Fischler, I.R. Klebanov and L. Susskind, hep-th/9709091.

39. I.R. Klebanov and L. Susskind, hep-th/9709108.

40. S.S. Gubser, I.R. Klebanov and A.W. Peet, Phys. Rev. D54 (1996) 3915, hep-th/9602135.

41. I.R. Klebanov and A.A. Tseytlin, Nucl. Phys. B475 (1996) 165, hep-th/9604089.

42. G. Horowitz and J. Polchinski, Phys. Rev. D55 (1997) 6189, hep-th/9612146.

43. L. Susskind, hep-th/9309145.

44. E. Witten, Nucl. Phys. B460 (1996) 541.

45. G. Horowitz and A. Strominger, Nucl. Phys. B360 (1991) 197.

46. S. Das, G. Gibbons and S. Mathur, Phys. Rev. Lett. 78 (1997) 417, hep-th/9609052.

47. R. Güven, Phys. Lett. B276 (1992) 49.

48. M.J. Duff and K.S. Stelle, Phys. Lett. B253 (1991) 113.

49. R. Emparan, hep-th/9706204.

50. A.A. Tseytlin, Nucl. Phys. B469 (1996) 51, hep-th/9602064.

51. S. Das, hep-th/9703146.

52. S.S. Gubser and I.R. Klebanov, hep-th/9708005.

53. D. Anselmi, M. T. Grisaru, and A. A. Johansen, hep-th/9601023.

54. D. Anselmi, D. Z. Freedman, M. T. Grisaru, and A. A. Johansen, hep-th/9608125.

55. U. Danielsson and G. Ferretti, "The Heterotic Life of the D-particle", hep-th/9610082.

56. G. Lifschytz, Phys. Lett. B388 (1996) 720, hep-th/9604156.

57. J. Polchinski and A. Strominger, Phys. Lett. B388 (1996) 736, hep-th/9510227.

58. O. Bergman, M. Gaberdiel and G. Lifschytz, "Branes, Orientifolds and the Creation of Elementary Strings", hep-th/9705130.

59. C.P. Bachas, M.R. Douglas and M.B. Green "Anomalous Creation of Branes", hepth/9705074.

60. T. Banks, N. Seiberg and E. Silverstein, Phys. Lett. B401 (1997) 30, hep-th/9703052. 